\title{
Relationship of Self-reported Attentional Fatigue to Perceived Work Ability in
}

\section{Breast Cancer Survivors}

Diane Von Ah; Susan Storey; Adele Crouch; Shelley A. Johns; Jill Dodson; Sarah Dutkevitch

Breast cancer survivors (BCS) have identified attentional fatigue as a frequent and troublesome symptom during and following treatment. ${ }^{1-4}$ The ability to pay attention is necessary to complete tasks and activities, and problem solve. Attention requires ongoing mental effort. For BCS, the prolonged or intense use of attention to address the competing mental demands associated with illness, treatment and daily life activties, can lead to attentional fatigue and result in decreased effectiveness in activity. ${ }^{5}$

Attentional fatigue, a domain of cognitive function, is often used interchangeably with the terms cognitive dysfunction, cognitive impairment, cognitive fatigue or tiredness. Seminal research, however, delineates attentional fatigue from these other concepts by describing attentional fatigue as a measurable decrease in an individual's ability to focus and concentrate or to block out distractions when carrying out meaningful activities. ${ }^{6}$ Hallmark indicators of attentional fatigue are: increased distractibility, difficulty following a train of thought or carrying out usual day-to-day activities, lapses in working memory, and increased impatience or frustration in personal interactions. ${ }^{7}$ Although the two may coexist, physical and attentional fatigue differ in that an individual may not be physically tired but has a reduced ability to exert mental effort. $^{8}$

Researchers have noted that BCS incur attentional fatigue more than age-matched controls ${ }^{9}$ and prostate cancer patients. ${ }^{10}$ Merriman and colleagues, ${ }^{11}$ found that up to $63 \%$ of

This is the author's manuscript of the article published in final edited form as:

Von Ah, D., Storey, S., Crouch, A., Johns, S. A., Dodson, J., \& Dutkevitch, S. (2016). Relationship of Self-reported Attentional Fatigue to Perceived Work Ability in Breast Cancer Survivors. Cancer Nursing, Publish Ahead of Print. https://doi.org/10.1097/NCC.0000000000000444 
breast cancer patients receiving radiation therapy reported moderate to high levels of attentional fatigue prior to and after treatment, suggesting that this is a prevalent and persistent problem for BCS. In the presence of attentional fatigue, rational decision making, social interactions and expression of emotions may be altered subsequently impacting work ability.

Attentional fatigue has been associated with poor outcomes in cancer survivors. One cross-sectional study of $134 \mathrm{BCS}$ found that higher levels of attentional fatigue were related to more depression and physical fatigue and to lower levels of physical functioning and psychological well-being. ${ }^{8}$ Additional evidence suggests that BCS with greater attentional fatigue also experience greater mood disturbance, ${ }^{8}$ depression, ${ }^{12,13}$ and overall poorer quality of life. ${ }^{12}$ However, little is known regarding the impact of attentional fatigue on work ability.

Perceived work ability, which includes the perceived physical, psychological, and social resources an individual has to participate in work, ${ }^{14,15}$ has been linked to overall well-being. ${ }^{16,17}$ In fact, for patients with cancer, work ability has been shown to contribute to meaning and purpose in life. ${ }^{16,17}$ Work ability is dependent on the mental and physical health of the individual in conjunction with social skills, level of education, motivation, work demands, and the organizational culture. ${ }^{14,17-19}$ Self-perceived work ability has been shown to be highly correlated with work performance, indicating that it is an important outcome measure..$^{20,21}$

In a study of 74 BCS, Boykoff and colleagues (2009) noted that BCS reported deficits in attention, such as the tendency to daydream, and also expressed concerns about decreased efficiency on the job. ${ }^{22}$ Similarly, Munir and colleagues noted that BCS $(N=13)$ reported difficulty in dividing attention among tasks, which affected their confidence in work ability. ${ }^{23}$ Although limited, these studies suggest that attentional fatigue may impact work ability in BCS. The challenges associated with work ability for BCS, many of whom are of working age, have 
yet to be fully explored. Further study is needed to understand the impact of attentional fatigue on the work ability of BCS. While there are multiple other factors that can contribute to attentional fatigue, this study attempted to examine the most salient factors (age, education, household income and time post treatment) noted in empirical research. Therefore, the purpose of this study was to examine the relationship between self-reported attentional fatigue and perceived work ability in BCS controlling for known confounding factors including age, education, household income, and time post-treatment.

The Cancer \& Work Model was used to guide this study. ${ }^{16}$ This comprehensive, empirically derived model depicts the consequences of cancer and/or associated post-treatment sequelae, including symptoms such as attentional fatigue, and their ongoing influence on workrelated outcomes such as work ability. ${ }^{16}$ The Cancer \& Work Model elucidates factors for both the clinician and survivor to consider that may impact workability and can be addressed by the health care provider, survivor and the work environment. ${ }^{16}$ For this study, we are adapted the model to specifically examine the relationship between attentional fatigue which falls under symptoms and perceived work ability which is identified as an outcome in the model. See Figure 1 for the conceptual framework. Our main interest was to advance the science by determining what, if any, association attentional fatigue has with perceived work ability over and above known confounding (demographic and medical characteristics) variables. Demographic characteristics of cancer survivors, including age and socio-economic status (education and household income), have been identified as influencing work ability ${ }^{15,16}$ Researchers have noted that younger women report more disruption from attentional fatigue, ${ }^{7,10}$ which could negatively impact work ability. The level of education may also affect work ability. Higher levels of education have been associated with better performance on the completion of complex tasks 
which require the ability to maintain attention. ${ }^{5}$ In addition, time post-treatment is an important factor influencing decisions related to work ability, with less time post-treatment associated with

poorer work ability. ${ }^{15,16,24}$ Studies have mainly focused on how age and socio-economic status impact return to work and/or work ability. Therefore, in this study we chose to examine the relationships of age, education, household income, time post-treatment and attentional fatigue on perceived work ability in BCS.

\section{Methods}

A cross-sectional, descriptive design was used. A convenience sample of BCS was recruited from a cancer center in the Midwestern United States. Eligible women were: (1) between the ages of 21 and 70 years of age, (2) employed full-time or part-time outside the home, (3) diagnosis of stage 0-III breast cancer and (4) at least 1 year post-adjuvant treatment (except for anti-hormonal agents). Patients were excluded if they: (1) brain metastasis, (2) history of stroke, brain injury, brain surgery, or dementia (rationale being that these could influence the severity and impact of attentional fatigue) or (2) were unemployed during the time of treatment.

\section{Procedure}

The study was approved by the university's institutional review board. Patients were screened for eligibility by clinic staff. Those expressing interest in participating in the study were asked to sign the informed consent and complete questionnaires eliciting demographic and treatment information, the Attention Function Index (AFI), and the Work Ability Index (WAI) and return the materials in a postage-paid envelope to the researcher. Upon receipt of these documents, participants were sent a $\$ 10$ gift card.

\section{Instruments}


Demographic and treatment characteristics were collected by the researcher.

Demographic information included: age, highest level of education, household income, gender, race, and marital status. Data regarding employment status (full-time or part-time hours) and type of occupation were also recorded. Participants were asked if they had changed either their occupation or employment status (full-time or part-time hours) after the diagnosis of breast cancer. Treatment characteristics included information on the date of diagnosis, location and stage (0-III) of the cancer, and type of treatment received (chemotherapy, surgery, radiation, tamoxifen, aromatase inhibitor).

Attentional fatigue was measured using the Attention Function Index(AFI), ${ }^{7}$ which is a 13-item ( 3 subscale) instrument that measures perceived effectiveness in common activities that require attention and working memory. ${ }^{7}$ The subscales measure effective action, attentional lapses and interpersonal effectiveness. ${ }^{7}$ The AFI was developed to assess the nefarious effects of cognitive or mental fatigue on the activities necessary for daily functioning. ${ }^{7}$ The total AFI score predicts mental fatigue. ${ }^{7}$ Higher scores indicate higher levels of attention. Scores of $>75$ indicate effective functioning, the moderate functioning range is $50-75$, and a score $<50$ is considered lower effectiveness or poor functioning. ${ }^{1}$ The AFI has established reliability and validity with a reported Cronbach's alpha of 0.92 for the total instrument and a range from 0.80 to 0.92 for the 3 subscales. ${ }^{7}$ For this study, Chronbach alpha for the AFI was .96.

Perceived work ability was measured using the Work Ability Index (WAI), which contains seven questions about the demands of work, the worker's health status, and resources. ${ }^{25}$ Response options are on a Likert-type scale from 1 to 7 . Total scores range from 7-49 points, and the following categories are determined by the score: poor work ability (7-27), moderate work ability (28-36), good work ability (37-43), and excellent work ability (44-49). Cronbach's 
alpha for the WAI has been reported as $0.72^{26}$ and test test reliability as $66 \% .{ }^{27}$ For this study, Chronbach alpha for the WAI was .89.

\section{Data Analysis}

Data were analyzed using the Statistical Package for the Social Sciences version 23. Descriptive statistics were generated for demographic, disease and treatment characteristics. To describe the sample, thresholds for the AFI and WAI were used. However, in the final analysis, the overall or total score for the AFI and WAI were used as a continuous variable to increase power in the linear regression model. Linear regression was chosen for this cross sectional study to examine the relationship between self-reported attentional fatigue and perceived work ability, controlling for the known covariates of age, education, household income, and time posttreatment. All of the variables were entered into the model at one time as in a prior study using AFI. ${ }^{8}$ An alpha level of 0.05 was used to test for statistical significance.

\section{Results}

Sixty-eight female BCS, who ranged from 29 to 68 years of age $(M=52.12 ; S D=8.60)$ and were on average $4.97(S D=3.36)$ years post-treatment, participated in the study. The BCS were primarily Caucasian, and married, had college degrees, and reported professional types of jobs and were employed full-time. Fifty $(75 \%)$ of the participants had been diagnosed with stage I or II breast cancer. Most of the participants $(67,98.5 \%)$ had received surgery for treatment. Forty-four (65\%) and forty-two (62\%) of the BCS had radiation and chemotherapy respectively. Thirty-five (52\%) of the women reported they had not taken Tamoxifen and forty-two (62\%) had noted they had not received aromatase inhibitors. Table 1 for the characteristics of the sample.

The total attentional fatigue scores for the BCS in this study ranged from 34 to 129 with a mean score of $84.34(S D=24.90)$. Categories for the AFI scores have been previously 
established and utilized by researchers. ${ }^{1,10,11}$ Of the BCS in this study, $47(69 \%)$ scored $>75$ on the AFI, with $11(16 \%)$ scoring in the 50-75 range and $10(15 \%)$ having scores of $<50$. Table 2 displays the AFI scores by category.

Perceived work ability scores for the BCS in this study ranged from 19 to $49(M=38.91$; $S D=7.45)$. Based on thresholds for this scale, over one-fourth $(26.5 \%)$ of BCS reported moderate to poor perceived work ability, indicating substantial concerns regarding work performance. Table 2 displays the WAI scores by category.

Linear regression results demonstrated a statistically significant relationship between attentional fatigue and perceived work ability $(p<0.001)$, explaining $40 \%$ of the variance in perceived work ability. Age, education, household income, and time post-treatment were not found to have a significant relationship with perceived work ability in this sample. See Figure 2 for linear regression results.

\section{Discussion}

The purpose of this study was to examine the relationship between self-reported attentional fatigue and perceived work ability in BCS. While there are multiple other factors that can contribute to attentional fatigue, this study attempted to examine the most salient factors noted in the empirical research and appropriate to the size of the sample. To our knowledge this is the first study to specifically examine attentional fatigue and work ability in this context. Several key findings were noted in this seminal study.

First, in this study, mean scores on the AFI were 84.34, indicating good functioning. However, a sub-population $(15 \%)$ of BCS in our sample scored $<50$ on the AFI, which indicates higher attentional fatigue and poorer functioning. Similarly, in a study of 73 BCS, researchers found that $22 \%$ of the women reported lower effectiveness and high levels of attentional fatigue 
$(\mathrm{AFI}<50) .{ }^{11}$ In a larger study of attentional fatigue, Cimprich and colleagues found a lower mean score on the AFI $(\mathrm{M}=65.31)$ in $172 \mathrm{BCS}$ prior to diagnostic biopsy and surgery (no percentages at each threshold level were reported). ${ }^{7}$ The difference in the AFI scores may be influenced by the timing of assessment in the illness trajectory, such as pre-treatment or during treatment versus post-treatment. Cimprich, Visovatti, and Ronis ${ }^{7}$ assessed attentional fatigue prior to the initiation treatment of treatment for early stage breast cancer. ${ }^{7}$ The early phases in the trajectory of breast cancer are times of uncertainty and stress, receipt of large amounts of new information, and decision making, often requiring intense attention. ${ }^{1,28} \mathrm{BCS}$ have found reported that changes in cognitive function, including symptoms of attentional fatigue may persist for years post diagnosis and treatment ${ }^{2.9,30}$ However, the duration, severity, and long term impact attentional fatigue yet to be explored among BCS. Although more research with larger sample sizes is needed to discern the prevalence and severity of attentional fatigue among BCS, these studies indicate that a substantial number of BCS incur attentional fatigue.

Second, over one-fourth of the 68 BCS in our study experienced moderate to poor work ability. These findings are significant since up to $90 \%$ of BCS return to work. ${ }^{31,32}$ Furthermore; work ability has been noted by BCS to contribute to a sense of normalcy and self-esteem, to be a distraction from the ongoing focus on cancer ${ }^{19,33}$ as well as to provide social networking and social support, giving meaning and purpose to their lives. ${ }^{33,34}$

Third, this was one of the first studies to fully examine the relationship between attentional fatigue and perceived work ability controlling for known covariates. Our study found that attentional fatigue was an important predictor of perceived work ability. The ability to focus attention requires continuous mental effort. When activities (such as those associated with working) require a prolonged capacity for attention, attentional fatigue may ensue. ${ }^{8}$ This study is 
the first to demonstrate a link between attentional fatigue and work ability using the full WAI and controlling for known covariates.

Our findings are supported by qualitative research. Fitch, Armstrong, and Tsang ${ }^{35}$ interviewed a heterogeneous sample of cancer survivors $(N=32)$, who reported that posttreatment changes in attention affected work ability. Other qualitative studies have reported similar findings. BCS $(N=12)$ have described attentional fatigue as restricting their work activity and work capacity. ${ }^{32}$ Cancer survivors $(N=29)$ reported that the inability to concentrate disrupted their work ability, negatively affected their productivity, and caused them to feel they were "letting [their employers] down." ${ }^{36}$ Quantitative studies are limited as the focus has been on cognitive function and work ability, not specifically on attentional fatigue. ${ }^{37-40}$

Lastly, our work provides initial support for our adaptation of the Cancer \& Work mode ${ }^{16}$ in that symptoms such as attentional fatigue may impact work ability in BCS. Working is beneficial to BCS; it provides structure and promotes self-esteem, distraction, social interaction, and financial stability. The work ability of cancer survivors is a global concern. As treatments for cancer continue to emerge and demonstrate effectiveness and as BCS live longer, they will make up a larger portion of the global workforce. Employers and health care providers need to be aware of potential problems such as attentional fatigue and its impact on work ability. In addition, employers need to be educated to identify and assist in addressing the specific needs of BCS to improve work ability.

\section{Implications for Nursing Practice}

Clinicians are in a key position to assess BCS for attentional fatigue and perceived work ability. Early identification of those who are experiencing attentional fatigue that impacts their work ability is important for both the BCS and employers. Research is needed to inform the 
development of comprehensive survivorship care plans and pre-emptive interventions to assist BCS with strategies to facilitate work ability.

The National Comprehensive Cancer Network $(\mathrm{NCCN})^{41}$ identifies strategies to address cognitive function in cancer survivors; however, there are very few evidence-based guidelines available to practitioners and survivors with tactics to address the disruptive symptom of attentional fatigue specifically. First-line intervention recommendations include: neuropsychological evaluation, cognitive therapy and physical activity. The use of psychostimulants is a second-line intervention. Strategies identified in a summary of qualitative studies in cancer survivors have supported these recommendations as well as identified selfmanagement activities used by BCS to mitigate symptoms. Self-management activities used by BCS include: validation of symptoms, self-organizing activities (note keeping, focusing on one thing at a time), mind stimulating activities (computer based training, crossword and word search puzzles), physical activity (exercise), adequate sleep, stress reduction (massage, meditation, yoga, and mindfulness based therapy ${ }^{42}$ In a recent study, BCS who received a mindfulness-based training intervention were assessed at 3 time points (baseline, post-intervention, and 6 months) and demonstrated a significant improvement in vitality and cancer-related fatigue. ${ }^{43}$ More research is needed to fully understand the impact of attentional fatigue on work-related outcomes in BCS. Future research should include mixed methods and/or longitudinal study designs that objectively measure attentional fatigue. In addition, mindfulness-based training interventions can be examined to assess its' impact on attentional fatigue and work ability.

\section{Limitations}

Study findings should be considered in terms of study limitations. The sample size was small and consisted of predominately well-educated Caucasian women, limiting its 
generalizability. The authors recognize there other factors that can affect attentional fatigue, but chose for this study to include and examine the most salient factors based on empirical research and the sample size. Lastly, we acknowledge the adapted model used in this study was a small portion of the comprehensive Cancer \&Work Model, nonetheless the findings provide initial evidence of a relationship between attentional fatigue and empirical support of the model. Future studies should include a larger and more diverse samples, be longitudinal in design, and should use objective measures of both attention and work-related outcomes (work ability, work performance and/or return to work). Studies exploring other prevalent, contributing factors to attentional fatigue and/or co-occurring, correlated symptoms or symptom clusters to understand fully the impact of symptoms on work ability in BCS are also needed.

\section{Conclusion}

Our findings indicate that attentional fatigue is a common post-treatment symptom that is negatively associated with perceived work ability in BCS. These findings are important and are clinically relevant for understanding the impact of cancer and cancer treatment on normal, daily activities such as work for BCS. This study suggests the need for individual, comprehensive survivorship care plans to effectively address symptoms that impact the quality of life of cancer survivors. 


\section{References}

1. Cimprich B, So H, Ronis DL, Trask C. Pre-treatment factors related to cognitive functioning in women newly diagnosed with breast cancer. Psychooncology. 2005;14(1):70-78.

2. Frank JS, Vance DE, Jukkala A, Meneses KM. Attention and memory deficits in breast cancer survivors: implications for nursing practice and research. J Neurosci Nurs. 2014;46(5):274-284 .

3. Von Ah D, Russell KM, Storniolo AM, Carpenter JS. Cognitive Dysfunction and Its Relationship to Quality of Life in Breast Cancer Survivors. Oncol Nurs Forum. 2009;36(3):326-336.

4. Wefel JS, Lenzi R, Theriault RL, Davis RN, Meyers CA. The cognitive sequelae of standard-dose adjuvant chemotherapy in women with breast carcinoma: results of a prospective, randomized, longitudinal trial. Cancer. 2004;100(11):2292-2299.

5. Cimprich, B., So, H., Ronis, D. L. \& Trask, C. (2005). Pre-treatment factors related to cognitive function in w omen newly diagnosed with breast cancer. Psychooncology 14, 70-78.

6. Cimprich B, Ronis DL. An environmental intervention to restore attention in women with newly diagnosed breast cancer. Cancer Nurs. 2003;26(4):284-292; quiz 293-284. 
7. Cimprich B, Visovatti M, Ronis DL. The Attentional Function Index--a self-report cognitive measure. Psychooncology. 2011;20(2):194-202.

8. Cimprich B. Attentional fatigue following breast cancer surgery. Res Nurs Health. 1992;15(3):199-207

9. Debess J, Riis JO, Pedersen L, Ewertz M. Cognitive function and quality of life after surgery for early breast cancer in North Jutland, Denmark. Acta Oncol. 2009;48(4):532540.

10. Merriman JD, Dodd M, Lee K, et al. Differences in self-reported attentional fatigue between patients with breast and prostate cancer at the initiation of radiation therapy. Cancer Nurs. 2011;34(5):345-353.

11. Merriman JD, Jansen C, Koetters T, et al. Predictors of the trajectories of self-reported attentional fatigue in women with breast cancer undergoing radiation therapy. Oncol Nurs Forum. 2010;37(4):423-432.

12. Von Ah D, Russell KM, Storniolo AM, Carpenter JS. Cognitive dysfunction and its relationship to quality of life in breast cancer survivors. Oncol Nurs Forum. 2009;36(3):326-336 .

13. Jansen CE, Dodd MJ, Miaskowski CA, Dowling GA, Kramer J. Preliminary results of a longitudinal study of changes in cognitive function in breast cancer patients undergoing chemotherapy with doxorubicin and cyclophosphamide. Psychooncology. 2008;17(12):1189-1195.

14. Short PF, Vasey JJ, Belue R. Work disability associated with cancer survivorship and other chronic conditions. Psychooncology. 2008;17(1):91-97. 
15. Moskowitz MC, Todd BL, Chen R, Feuerstein M. Function and friction at work: a multidimensional analysis of work outcomes in cancer survivors. J Cancer Surviv. 2014;8(2):173-182.

16. Feuerstein M, Todd BL, Moskowitz MC, et al. Work in cancer survivors: a model for practice and research. J Cancer Surviv. 2010;4(4):415-437.

17. Islam T, Dahlui M, Majid HA, et al. Factors associated with return to work of breast cancer survivors: a systematic review. BMC Public Health. 2014;14 Suppl 3:S8.

18. Ilmarinen J. Aging Workers. J Occup Environ Med. 2001;58:546-552.

19. Wells M, Williams B, Firnigl D, et al. Supporting 'work-related goals' rather than 'return to work' after cancer? A systematic review and meta-synthesis of 25 qualitative studies. Psychooncology. 2013;22(6):1208-1219.

20. Sell L, Bultmann U, Rugulies R, Villadsen E, Faber A, Sogaard K. Predicting long-term sickness absence and early retirement pension from self-reported work ability. Int Arch Occup Environ Health. 2009;82(9):1133-1138.

21. Kuoppala J, Lamminpaa A, Vaananen-Tomppo I, Hinkka K. Employee well-being and sick leave, occupational accident, and disability pension: a cohort study of civil servants. J Occup Environ Med. 2011;53(6):633-640.

22. Boykoff N, Moieni M, Subramanian SK. Confronting chemobrain: An indepth look at survivors' reports of impact on work, social networks, and health care response. J Cancer Surviv. 2009;3(4):1932-2267.

23. Munir F, Burrows J, Yarker J, Kalawsky K, Bains M. Women's perceptions of chemotherapy-induced cognitive side affects on work ability: a focus group study. J Clin Nurs. 2010;19(9-10):1362-1370. 
24. Steiner JF, Cavender TA, Main DS, Bradley CJ. Assessing the impact of cancer on work outcomes: what are the research needs? Cancer. 2004;101(8):1703-1711.

25. Ilmarinen J. The Work Ability Index (WAI). Occupational Medicine-Oxford. 2007;57(2):160-160.

26. Radkiewicz P, Widerszal-Bazyl M. Psychometric properties of Work Ability Index in the light of comparative survey study. International Congress Series. 2005;1280:304-309.

27. de Zwart BC, Frings-Dresen MH, van Duivenbooden JC. Test-retest reliability of the Work Ability Index questionnaire. Occup Med (Lond). 2002;52(4):177-181.

28. Cimprich B. Pretreatment symptom distress in women newly diagnosed with breast cancer. Cancer Nurs. 1999;22(3):185-194; quiz 195.

29. Koppelmans, V, Bretler, M, Boogerd, MM, Seynaeve, W, Gundy, C, Schagen, SB. Neuropsychological performance in survivors of breast cancer more than 20 years after adjuvant chemotherapy. J Clin Oncol. 2012;30(10):1080-1086.

30. Von Ah, D, Harvison, K, Monahan, P, Moser, L, Zahn, Q, ....Carpenter, J. Cognitive function in breast cancer survivors compared to healthy age-and education matched women. Clin Neuropsychol. 2009; 23 (4), 661-674.

31. Ahn E, Cho J, Shin DW, et al. Impact of breast cancer diagnosis and treatment on workrelated life and factors affecting them. Breast Cancer Res Treat. 2009;116(3):609-616.

32. Tamminga SJ, de Boer AG, Verbeek JH, Frings-Dresen MH. Breast cancer survivors' views of factors that influence the return-to-work process--a qualitative study. Scand $J$ Work Environ Health. 2012;38(2):144-154. 
33. Johnsson A, Fornander T, Rutqvist LE, Olsson M. Factors influencing return to work: a narrative study of women treated for breast cancer. Eur J Cancer Care (Engl). 2010;19(3):317-323.

34. Banning M. Employment and breast cancer: a meta-ethnography. Eur J Cancer Care (Engl). 2011;20(6):708-719.

35. Fitch MI, Armstrong J, Tsang S. Patients' experiences with cognitive changes after chemotherapy. Can Oncol Nurs J. 2008;18(4):180-192.

36. Kennedy F, Haslam C, Munir F, Pryce J. Returning to work following cancer: a qualitative exploratory study into the experience of returning to work following cancer. Eur J Cancer Care (Engl). 2007;16(1):17-25.

37. de Boer AG, Verbeek JH, Spelten ER, et al. Work ability and return-to-work in cancer patients. Br J Cancer. 2008;98(8):1342-1347.

38 Nieuwenhuijsen K, de Boer A, Spelten E, Sprangers MA, Verbeek JH. The role of neuropsychological functioning in cancer survivors' return to work one year after diagnosis. Psychooncology. 2009;18(6):589-597.

39. Taskila T, Martikainen, R., Hietanen, P., Lindbohm, M. Comparative study of work ability between cancer survivors and their referents. Eur J Cancer. 2007;43(5):914-920.

40. Torp S, Nielsen RA, Gudbergsson SB, Dahl AA. Worksite adjustments and work ability among employed cancer survivors. Support Care Cancer. 2012;20(9):2149-2156.

41. Denlinger, CS, Ligibel, JA. Are, M., Baker, KS, Demark-Wahnefried, W, Friedman, D L....Montoya, J G. Survivorship:Cognitive Function Version 1.2014. J Natl Compr Canc Netw.2014; 976-985

42. Von Ah, D, Storey, S, Jansen, CE \& Allen, DH. Coping strategies and interventions 
for cognitive changes in patients with cancer. Semin Oncol Nurs. 2013; 29(4), 288-299.

43. Johns, S.A., Von Ah, D., Brown, L.F., Beck-Coon, K., Talib, T.L., Alyea, J.M., Monahan, P.O., Tong, Y., Wilhelm, L., Giesler, R.B. Randomized controlled pilot trial of Mindfulness-Based Stress Reduction for breast and colorectal cancer survivors: Effects on cancer-related cognitive impairment. Journal of Cancer Survivorship: Research and Practice. 2016; 10(3), 437-448. 
Figure 1. Conceptual Framework

\begin{tabular}{|c|}
\hline \multicolumn{1}{|c|}{ Antecedents } \\
Demographic characteristics \\
- Age \\
- Education \\
- Household income \\
\hline
\end{tabular}

\section{Medical characteristic}

- Time post treatment

\section{Attentional fatigue}

- Attention Function Index (AFI)

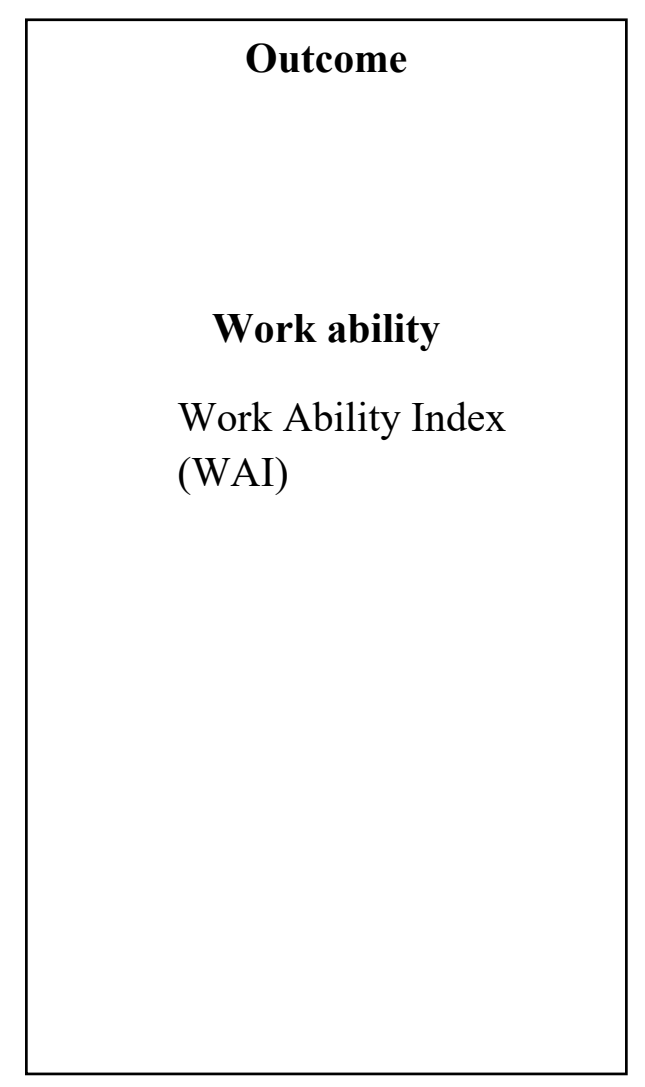


Table 1 Sample Characteristics $(N=68)$

\begin{tabular}{|c|c|c|}
\hline & $\underline{\text { Mean }}(\underline{\mathrm{SD}})$ & $\underline{\mathrm{n}}(\underline{\%})$ \\
\hline$\overline{\text { Age }}$ & $52.12(8.603)$ & \\
\hline \multicolumn{3}{|l|}{ Race } \\
\hline African American & & $4(5.9)$ \\
\hline Caucasian & & $63(92.6)$ \\
\hline Missing & & $1(1.5)$ \\
\hline \multicolumn{3}{|c|}{ Highest Level Education Completed } \\
\hline $1-11$ & & $0(0)$ \\
\hline 12 (High School) & & $12(17.6)$ \\
\hline 13 & & $4(5.9)$ \\
\hline 14 & & $6(8.8)$ \\
\hline 16 (College) & & $23(33.8)$ \\
\hline 17 & & $2(2.9)$ \\
\hline 18 (Master's degree) & & $17(25)$ \\
\hline 19 & & $1(1.5)$ \\
\hline 20 (doctorate) & & $1(1.5)$ \\
\hline Missing & & $2(2.9)$ \\
\hline \multicolumn{3}{|l|}{ Employment Status } \\
\hline Employed Full time & & $51(75)$ \\
\hline Employed Part time & & $14(20.6)$ \\
\hline Missing & & $3(4.4)$ \\
\hline
\end{tabular}


Type of Employment

Professional (law, medicine, teacher, engineer) $29(42.6)$

Management/Administration $\quad 11(16.2)$

Technical (repairman, computer skills) $3(4.4)$

Clerical (secretary) $13(19.1)$

Service (waiter/waitress, sales) 3 (4.4)

Self-employed $1(1.5)$

Other $\quad 6(8.8)$

Missing $2(2.9)$

Marital Status

$\begin{array}{ll}\text { Single } & 10(14.7)\end{array}$

Single/Living with partner $\quad 5(7.4)$

Married 48 (70.6)

Widowed $1(1.5)$

Other $3(4.4)$

Missing $1(1.5)$

Income
$<\$ 15,000$
$1(1.5)$
$\$ 15,001$ to $\$ 30,000$
$4(5.9)$
$\$ 30,001$ to $\$ 50,000$
$5(7.4)$
$\$ 50,001$ to $\$ 75,000$
$5(7.4)$
$\$ 75,001$ to 100,000
$25(36.8)$
$\$ 100,001$ to $\$ 150,000$
$10(14.7)$ 
$\$ 150,001$ to $\$ 200,000$

$>\$ 200,000$

Don't know

Missing
$11(16.2)$

$3(4.4)$

$1(1.5)$

$3(4.4)$ 
Table 2. Attentional Function Index (AFI) and Work Ability Index (WAI) Scores

\begin{tabular}{|c|c|c|c|c|}
\hline AFI & $n$ & Mean (SD) & Minimum & Maximum \\
\hline Overall score & 68 & $84(25)$ & 34 & 129 \\
\hline Threshold & $\mathrm{n}(\%)$ & & & \\
\hline $\begin{array}{l}\text { Effective } \\
\text { functioning }>75\end{array}$ & $45(66)$ & $99.2(14.0)$ & 81 & 129 \\
\hline $\begin{array}{l}\text { Moderate } \\
\text { functioning } \\
50-75\end{array}$ & $14(21)$ & $63.6(6.0)$ & 52 & 72 \\
\hline $\begin{array}{l}\text { Lower } \\
\text { functioning }<50\end{array}$ & $9(13)$ & $43.5(5.0)$ & 34 & 49 \\
\hline
\end{tabular}

\begin{tabular}{|c|c|c|c|c|}
\hline WAI & $n$ & Mean (SD) & Minimum & Maximum \\
\hline Overall score & 68 & $38.9(7.0)$ & 19 & 49 \\
\hline Threshold & $n(\%)$ & & & \\
\hline $\begin{array}{l}\text { Excellent } \\
(44-49)\end{array}$ & $19(28)$ & $46.6(1.7)$ & 44 & 49 \\
\hline $\begin{array}{l}\text { Good } \\
(37-43)\end{array}$ & $31(46)$ & $40.4(1.9)$ & 37 & 43 \\
\hline Moderate & $11(16)$ & $31.8(1.9)$ & 29 & 34 \\
\hline
\end{tabular}




\begin{tabular}{|l|l|l|l|l|}
\hline$(28-36)$ & & & & \\
\hline Poor & $7(10)$ & $23.4(3.0)$ & 11 & 27 \\
$(7-27)$ & & & & \\
\hline
\end{tabular}

